\title{
28 Research Square \\ Identification and Bioinformatics Analysis of \\ Differentially Expressed Milk Exosomal Micrornas in Milk Exosomes of Heat-Stressed Holstein Cows
}

\section{Yue Wang}

Nanjing Agricultural University

Jian Fang

Nanjing Agricultural University

Han-Fang Zeng

Nanjing Agricultural University

Hui-Xia Li

Nanjing Agricultural University

Kun-Lin Chen ( $\nabla$ chenkunlin@jaas.ac.cn )

Jiangsu Academy of Agricultural Sciences https://orcid.org/0000-0002-8398-6922

\section{Research}

Keywords: miRNAs, Milk exosomes, Heat stress, Apoptosis, MAPK signaling pathway, Small RNA sequencing

Posted Date: June 16th, 2021

DOI: https://doi.org/10.21203/rs.3.rs-593653/v1

License: (c) (1) This work is licensed under a Creative Commons Attribution 4.0 International License. Read Full License

Version of Record: A version of this preprint was published at Functional \& Integrative Genomics on November 27th, 2021. See the published version at https://doi.org/10.1007/s10142-021-00814-8. 


\section{Abstract \\ Background}

In summer, heat stress is one of the primary reasons for the compromised health and low milk productivity of dairy cows. Hyperthermia affects milk synthesis and secretion in the mammary glands of dairy cows. As molecules for intercellular communication, milk-derived exosomes carry genetic material, proteins and, lipids, playing a crucial role in mammary tissue growth and milk synthesis in dairy cows. The aim of this study was to explore the milk exosomal miRNAs profile of heat-stressed and normal Holstein cows.

\section{Results}

We isolated and identified milk exosomes to screening for differentially expressed miRNAs using small RNA sequencing. Then, TargetScan and miRanda algorithms were used to predict the putative targets of the differentially expressed miRNAs, whereas GO and KEGG pathway enrichment analyses were performed for the differentially expressed miRNA-target genes. Our results showed that 215 miRNAs were significantly differentially expressed in heat-stressed milk exosomes, of which one was up-regulated and 214 were significantly downregulated. GO and KEGG enrichment analyses indicated that differentially expressed miRNAs might play a role in apoptosis, autophagy, and the p38 MAPK pathway. qRT-PCR assay verified that the expression of miRNAs was consistent with the sequencing results, warranting further verification of their specific targets of action.

\section{Conclusions}

In conclusion, changes in the miRNA expression profile of milk exosomes indicated the role of exosomal miRNAs in regulating heat stress resistance and apoptosis in dairy cows. Our results suggested that milkderived exosomal miRNAs could increase mammary gland resistance to heat stress, thereby enhancing milk synthesis in dairy cows.

\section{Background}

Heat stress (HS) refers to a metabolic disorders in animals caused by an increase in ambient temperature, which causes damage to animal tissues and organs [1]. In the livestock industry, HS is a key concern because it seriously influences not only reproductive performance but also milk yield [2]. Bovine mammary epithelial cells (BMECs) are the secretory cells of the mammary glands, which play an important role in milk synthesis. HS reduces the ability of BMECs to synthesize and secrete milk, further affecting the quality and yield of milk. Our previous study showed that HS triggers apoptosis in BMECs and causes changes in their morphology, structure, and function [3-5]. Although the mechanism of HSinduced injury of BMECs has been lucidated, the role of exosomal miRNAs in the HS-regulating 
mechanism of BMECs is still unclear. Therefore, this study aimed to investigate the miRNA profiles of milk exosomes of heat-stressed Holstein cows.

Milk contains a large number of functional substances, including proteins, lipids, and exosomes, which are vital nutrients needed for a wide array biological activities [6]. Milk synthesis in dairy cows is often affected by many environmental, physical, and chemical factors. It has been reported that during an inflammatory response, milk-derived exosomes are mediated by the TLR4/NF-KB and p53 pathways to reduce $B M E C$ apoptosis and protect LPS-induced intestinal epithelial cells against apoptosis [7]. Porcine milk components, including exosomes, are associated with mammary gland function [8]. Zhang et al. showed that exosomal proteins are closely related to the biosynthesis of milk by BMECs [9]. It is generally believed that RNA is degraded by RNases in the environment. However, milk exosomes can prevent miRNA degradation [10]. Exosomes can be directly absorbed in the small intestine upon gastric acid stimulation, conferring a protective effect on the immune system and the health of neonates [11]. Moreover, exosomes can be used as carriers to deliver various macromolecules into target cells without triggering an immune response [12].

Exosomes (Exo) are tiny membranous vesicles secreted by cells. They are composed of a lipid bilayer membrane with a vesicle diameter of $20-150 \mathrm{~nm}$ and enriched in proteins, lipids, and nucleic acids; in addition, they act as signaling molecules, regulating many types of cellular functions [13]. After their entry via endocytosis or membrane receptors, exosomes release proteins, mRNAs, miRNAs, and IncRNAs into the target cells, thereby changing the gene expression profile of these cells [14]. miRNAs control gene expression by degrading mRNA and inhibiting translation $[15 ; 16]$. Exosomes derived from mesenchymal stem cells can protect $\beta$ cells from hypoxia-induced apoptosis, reduce cellular stress, and inhibit the activation of the p38 MAPK signaling pathway [17]. miR-100-5p in human umbilical cord MSC-derived exosomes protects cardiomyocytes from hypoxic injury and inhibits inflammasome activation-mediated pyroptosis [18]. Qiu et al. have shown that exosomes carrying miR-181a-3p plays a critical role in apoptosis by alleviating endoplasmic reticulum stress [19]. In addition, the study showed that bovine milk exosomes enhanced the viability and antioxidant capacity of intestinal cryogenic epithelial cells, and exosome pretreatment increases intracellular miR-146a and miR-155 levels [20]. When the integrity of BMECs was compromised, the health and milk yield of dairy cows can be highly affected. Previous studies have shown that miRNAs are not only related to apoptosis but involved in the physiological processes of breast development and milk synthesis [21]. In line with this, Hou et al. reported that 5, 10methylenetrahydrofolate reductase modulates goat milk synthesis by regulating the binding activity of miR-1266 and miR-616 [22].

In the present study, we aimed to identify significantly differentially expressed miRNAs in exosomes derived from heat-stressed Holstein cow milk using small RNA sequencing technology and to predict the target gene and possible pathways associated with stress and injury. Our results suggested that the expression profile of milk exosomal miRNAs could regulate the development and function of mammary tissue in heat-stressed dairy cows via the MAPK pathway. Our findings serve as a new reference for studies on alleviating HS in cows during summer. 


\section{Methods}

\section{Sample collection}

Continuous monitoring of temperature and humidity index (THI) of cowshed, rectal temperature and respiratory rate of China Holstein cows under the same management level in summer (August, heat stress group (HS)) and winter (December, control group (Ctr)) in SiHong ranch of JiangSu province, China. 50mL milk from 6 Holstein cows with similar lactation periods and parity in the HS group and Ctr group were collected, transported to the laboratory at low temperature and stored at $-80^{\circ} \mathrm{C}$ for subsequent sequencing. The data of $\mathrm{THI}$, rectal temperature and respiratory rate are shown in Table 1.

\section{Extraction and identification of exosomes}

The frozen milk sample was heated in a water bath at $37^{\circ} \mathrm{C}$. After dissolution, the sample was added to a new centrifuge tube, and centrifuged at $4^{\circ} \mathrm{C}$ for $30 \mathrm{~min}$ at $2,000 \mathrm{~g}$. The supernatant was transferred to a new centrifuge tube at $10,000 \mathrm{~g}$ at $4^{\circ} \mathrm{C}$ for $45 \mathrm{~min}$. The supernatant was centrifuged at $100,000 \mathrm{~g}$ for $120 \mathrm{~min}$ at $4^{\circ} \mathrm{C}$ in a new centrifuge tube. The supernatant was discarded and resuspended with $20 \mathrm{~mL}$ precooled $1 \times$ PBS, centrifuged at $2,000 \mathrm{~g}$ for $30 \mathrm{~min}$ at $4^{\circ} \mathrm{C}$. Supernatant was centrifuged at $4^{\circ} \mathrm{C}$ of $2,000 \mathrm{~g}$ for $30 \mathrm{~min}$. The centrifugation was repeated at $2,000 \mathrm{~g}$ at $4^{\circ} \mathrm{C}$ for $30 \mathrm{~min}$. Then the supernatant was centrifuged at $100,000 \mathrm{~g}$ at $4^{\circ} \mathrm{C}$ for $120 \mathrm{~min}$. The supernatant was discarded, resuspended and precipitated with $1 \mathrm{~mL}$ precooled PBS, and temporarily stored at $4{ }^{\circ} \mathrm{C} .40 \%, 20 \%, 10 \%$ and $5 \%$ iodoxanol were prepared. lodoxanol of different concentrations was added along the tube wall (3.6mL for each) according to the high to low concentration. Finally, $1 \mathrm{~mL}$ resuspend temporarily stored at $4{ }^{\circ} \mathrm{C}$ was added to the top layer at $4^{\circ} \mathrm{C}, 100,000 \mathrm{~g}$ for $120 \mathrm{~min}$. After centrifugation, it was divided into 12 layers, the liquid in the middle 6-9 layers was taken out, and centrifuged again at $4^{\circ} \mathrm{C}$ for $100,000 \times \mathrm{g}$ for $120 \mathrm{~min}$. The supernatant was removed, and the exosomes were resuspend with $1 \times \mathrm{PBS}$ precooled with $100 \mu \mathrm{L}$ stored at $-80^{\circ} \mathrm{C}$.

\section{Transmission electron microscopy}

$10 \mu \mathrm{L}$ of exosomes was absorbed and added to the copper net precipitation for $1 \mathrm{~min}$, the floating solution was removed by filter paper, and the phosphotungstic acid was added to the copper net precipitation for $1 \mathrm{~min}$. The floating solution was removed by filter paper and dried for several minutes at room temperature. The transmission electron microscopy imaging was performed at $100 \mathrm{kv}$.

\section{Nanoparticle tracking analysis}

The samples were thawed in a water bath at $25^{\circ} \mathrm{C}$, and placed on ice. Exosome samples were diluted with $1 \times$ PBS and directly used for NTA detection.

\section{Western Blotting}


The exosomes were centrifuged at $10,000 \mathrm{~g}$ at room temperature for $5 \mathrm{~min}$, then supernatant was

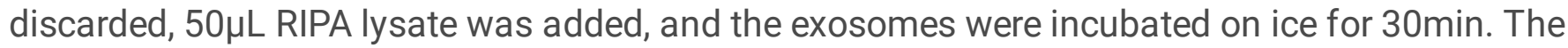
concentration of exosomes was determined by BCA kit, and denaturated in metal bath at $100^{\circ} \mathrm{C}$ for $10 \mathrm{~min}$; and then SDS-PAGE was performed. The protein was transferred to PVDF membrane by wet transfer method, and was sealed with $5 \%$ skim milk for $1 \mathrm{~h}$. Primary antibodies CD81, CD63, TSG101 and Calnexin (1:1000) polyclonal antibodies were added and incubated overnight at $4^{\circ} \mathrm{C}$. Washing with 1 IITBST buffer for 3 times, $5 \mathrm{~min}$ each time, then add HRP-labeled secondary antibody and incubate at room temperature for $90 \mathrm{~min}$. After washing with $1 \times$ TBST buffer for 3 times, $5 \mathrm{~min}$ each time, ECL chemiluminescence was developed and scanned in the infrared imaging system.

\section{Small RNA sequencing and analysis}

Total RNA was extracted from exosomes, and the Small RNA sequencing library was prepared by Truseq Small RNA Sample Prep Kits (Illumina, San Diego, USA). In simple terms, the total RNA was added with 3 'and 5 ' adapter, and the Small RNA connected by the adapter was used for reverse transcription PCR to create the DNA library, and the primer was annealed to connect the tow fringes adapter for PCR, and the Small RNA library was purified by gel. Finally, the High Sensitivity DNA Chip was used to test the sample library. The qualified libraries were sequenced using Illumina Hiseq2000/2500 with a single-ended read length of 7 í $50 \mathrm{bp}$.

\section{Screening of differentially expressed miRNAs}

Raw reads were subjected to ACGT101-miR (LC Sciences, Houston, Texas, USA) to remove adapter dimers, junk, low complexity, common RNA families (rRNA, tRNA, snRNA, snoRNA) and repeats. The final data is the valid reads, which can be used for subsequent analysis of small RNA data. According to the data of miRNAs expression in each sample, $T$ test was used to analyze the differential expression of miRNAs, and according to the significance of expression difference $(P<0.05)$ differentially conserved miRNAs were screened out.

\section{Target gene prediction and GO and KEGG enrichment analysis}

miRNAs bind to target sites mainly through complementary pairing. The 3 ' UTR sequence of bovine mRNA was used as the target sequence to predict the target genes of differentially expressed miRNAs sequences. We used TargetScan (v5.0) and miRanda (v3.3a) software to predict the target genes of miRNAs with significant differences. The target genes were screened predicted with two software according to the scoring criteria. Target genes with the context score percentile less than 50 were removed in the TargetScan algorithm, and target genes with the maximum free Energy (Max Energy) >-10 were removed in the miranda algorithm (that is, the threshold was targetScan_score $\geq 50$, miranda_Energy <-10). Finally, the intersection of software was selected as the final target gene of the differential miRNAs. 
The GO functional significance enrichment analysis first mapped all the differentially expressed genes to each term in the Gene Ontology database (ftp://ftp.ncbi.nih.gov/gene/DATA/gene2go.gz), calculated the number of genes in each term, and then applied hypergeometric test to find the $\mathrm{GO}$ items significantly enriched in differentially expressed genes compared with the entire genome background. KEGG is the main public database on Pathways. Pathway significance enrichment analysis uses KEGG Pathway (http://www.genome.jp/kegg) as the unit, and hypergeometric tests are used to identify the pathways that are significantly enriched in differentially expressed genes compared with the overall genome background.

\section{Quantitative real-time PCR (qRT-PCR)}

Total RNA was extracted with TRIzol ${ }^{\mathrm{TM}}$ reagent (Invitrogen, cat: 15596026, USA). Before exosomes RNA extraction, synthetic cel-miR-39 (GenePharma, Shanghai) was added as an internal control [23]. The density of samples RNA was quantified spectrophotometrically at 260/280 nm. miRNA 1 st Strand cDNA Synthesis Kit (by stem-loop) (Vazyme, cat: MR101-01/02, Nanjing) was used to reverse transcribe the total RNA, and mRNA expression was quantified with real-time PCR. Using comparative Ct $\left(2^{-\Delta \Delta C t}\right)$ value method standardizes the expression levels of all target genes with the endogenous reference gene U6 or Cel-miR-39 (Forward primer: ATATCATCTCACCGGGTGTAAATC; Reverse primer: TATGGTTTTGACGACTGT

GTGAT). The primers sequences are listed in supplementary materials Table S1.

\section{Statistical analysis}

Dates are shown as mean values \pm SEM. All results were analyzed by $T$ test, using the software GraphPad Prism 8.0.1 (La Jolla, CA, USA). $P<0.05$ was considered statistically significant.

\section{Results}

\section{Identification of milk exosomes}

Exosomes originate from the multi-vesicles in living cells and are secreted as membranous vesicles, with a density of $8.76 \mathrm{E}+9$ particles/mL and a typical "cup and plate" shape (Fig. 1A, C-D). TEM revealed that milk exosomes negatively stained with phosphotungstic acid had a diameter of approximately $100 \mathrm{~nm}$. NTA showed that the average particle size of exosomes was $73.82 \mathrm{~nm}$, and the size of exosomes in the range of $30-150 \mathrm{~nm}$ accounted for $97.8 \%$ of the all particles (Fig. 1E-F). CD81, CD63, and TSG101 proteins are the signature proteins of exosomes, and their presence further proves that the vesicles observed using an electron microscope are exosomes. Calnexin is a protein on the endoplasmic reticulum, which acts as a molecular chaperone to participate in the folding and processing of new peptide chains of proteins. Our western blotting results showed that proteins CD81, CD63, and TSG101, except calnexin, were expressed in exosomes compared to breast epithelial cells, indicating that the extracted exosomes were pure and not contaminated (Fig. 1B). 


\section{Sequencing quality analysis and miRNAs length distribution}

To detect the expression of miRNAs in milk exosomes of cows under HS and normal conditions, the single-ended $1 \times 50$ bp sequencing reading length of Illumina HiSeq2000/2500 platform was used for exosome detection. A total of six exosome samples were detected, including three each from the HS group (HS1, HS2, and HS3) and non-HS group (Ctr1, Ctr2, and Ctr3). The total read length of the original sequencing was 133884666 , and the average read length of each sample was 22314111 . Initially, in the original sequencing data, the 3 ' connector sequence was removed, and the sequences with base length less than $18 \mathrm{nt}$ were removed. Eighty percent of the sequences were $\mathrm{A}, \mathrm{C}, \mathrm{G}$, or $\mathrm{T} ; 3 \mathrm{~N}$ (not necessarily continuous); only $\mathrm{A}$ and $\mathrm{C}$ without $\mathrm{G}$ or $\mathrm{T}$; or only $\mathrm{G}$ and $\mathrm{T}$ without $\mathrm{A}$ or $\mathrm{C}$, and continuous nucleotide dimers, and trimers were removed (Table 2).

Based on the original sequencing data, we conducted length distribution statistics on the filtered data. Most of the sequences were 20-24 nt in length, indicating the typical characteristics of a Dicer enzyme cleavage (Fig. 2A). We compared the measured sequences to mRNA, RFam (including rRNA, tRNA, snRNA, and snoRNA) and the Repbase database to yield sequencing data that can be considered data (Table 3). Subsequently, miRNA comparison, identification, and prediction analyses were performed on the valid sequencing data, and a total of 42159649 valid reads were identified. Further analysis showed that $80.67 \%$ of all the screened sequences were miRNAs, while $6.56 \%, 10.43 \%, 0.21 \%, 0.32 \%$ and $1.80 \%$ of the total sequencing data belonged to rRNA, tRNA, snoRNA, snRNA, and other RFam RNAs (Fig. 2B). In addition, we compared the differentially expressed miRNAs on chromosomes, and the generated circle polt showed that miRNAs were evenly distributed on each chromosome (Fig. 2C).

\section{Differential expression analysis of miRNAs}

Differential expression of 215 miRNAs was detected in the screened sequencing data $(P<0.05)$ (Table S2), among which 208 were known and seven were novel miRNAs, compared to the precursors of the selected species in miRBase (Fig. 3A, Tables S3-S4). Compared to the Ctr groups, the expression of one miRNA was significantly upregulated, while that 214 miRNAs were significantly downregulated (Fig. 3B, Table S2). In addition, significantly different expression of 215 miRNAs was predicted for 12,446 target genes (Table S5). bta-miR-15a had the highest number of target genes. Among them, the most significantly enriched target gene with the most number of differentially miRNAs was UBE2W (66 miRNAs).

\section{GO and KEGG enrichment analyses for differentially expressed miRNAs}

We performed GO analysis to identify the biological processes associated with the identified miRNAs (Fig. 4A). These differentially expressed miRNA target genes were significantly enriched in 1,432 GO pathways $(P<0.05)$, among which $910 \mathrm{GO}$ pathways were associated with biological processes, 245 were cellular components, and 277 were molecular functions (Table S6). Moreover, the target genes were significantly enriched in 188 KEGG pathways (Table S7). Among the top 20 significantly enriched KEGG pathways, the pathway for cancer $(P=2.02 \mathrm{E}-09)$ was the most significantly enriched (Fig. 4B). 


\section{Bioinformatics analysis of differentially expressed miRNAs}

Nine of the top fifty miRNAs were differentially expressed with the highest degree of enrichment in in the two groups. These nine miRNAs were closely related to autophagy, apoptosis, and the MAPK signaling pathways (Fig. 5A). To examine the association between differentially expressed milk exosomal miRNAs and their target genes, miRNA-gene interaction networks were generated using Cytoscapses (Fig. 5B, Table S8). Network analysis revealed results consistent with KEGG pathway prediction for the nine miRNAs, including the MAPK signaling pathway.

qRT-PCR results showed that the expression of bta-miR-339a_R-2, bta-miR-339b, chi-miR-340-5p, bta-miR141_R-1, bta-miR-30b-5p, bta-miR-30f_R-3, bta-miR-27a-3p, and bta-miR-20a in exosomes and BMECs was decreased, whereas that of bta-miR-193a-5p was increased in heat-stressed cows (Fig. 6).

\section{Discussion}

HS not only damages the health of cows but also affects milk synthesis. Milk is rich in various bioactive substances, including lipids, proteins, and exosomes, which are crucial for biological activities. Milk exosomal proteins and miRNAs play a key role in regulating cell proliferation, inflammatory responses, immune regulation, and cancer cell proliferation [24-26]. Based on these functions of milk-derived exosomes, we sought to differentially expressed miRNAs in exosomes derived from the mil of heatstressed Holstein cows using small RNA sequencing technology, and we predicted the target genes and possible enriched pathways involved in stress response and apoptosis.

The mechanism by which mRNA, miRNAs and IncRNAs in milk-derived exosomes participate in the physiological processes of cells is still unclear. miRNAs play an important role in cellular communication as signal transduction molecules [27]. Smythies et al. showed that stress can change the content of exosomal miRNA profiles of developing sperms in the epididymis [28]. In this study, we isolated and validated the exosomes derived from the milk of heat-stressed cows. High-throughput sequencing analysis showed that $80.67 \%$ of all screened sequences were miRNAs. We detected 215 differentially expressed miRNAs in the screened sequencing data, including one miRNA that was significantly upregulated, and 214 miRNAs, which were significantly downregulated. Therefore, milk exosomal miRNAs have different expression profiles in the cows of the control and HS groups. Differential miRNA expression is often accompanied by changes in cellular function [29]. GO analysis identified the biological processes associated with the differentially expressed miRNA target genes enriched in 1,432 pathways $(P<0.05)$, among which $910 \mathrm{GO}$ pathways were associated with biological processes, 245 were cellular components, and 277 were molecular functions. KEGG pathway enrichment analysis that the differentially expressed miRNAs mainly play a role in the MAPK signaling pathway, endotosis, human T-cell leukemia virus 1 infection, the Rap1 signaling pathway and rheumatoid arthritis, which have been shown to be closely related to apoptosis [30;31]. Therefore, we speculated that differential expression of milk exosomal miRNAs could play an important regulatory in the injury response of heat-stress dairy cows. 
Compared to the Ctr group, the top 10 differentially expressed of miRNAs included bta-miR-339a_R-2, btamiR-339b, chi-miR-340-5p, bta-miR-141_R-1, bta-miR-30b-5p, bta-miR-30f_R-3, bta-miR-27a-3p, and btamiR-193a-5p in the HS group were significantly downregulated, and bta-miR-193a-5p, which was significantly upregulated. Previous studies have shown that miR-27a-3p via BTG1 to regulate apoptosis in the colon cancer cell line, HCT-116, via the ERK/MEK pathway [32]. Li also demonstrated that miR-27a$3 p$ regulates cellular inflammation and autophagy [33]. Function prediction analysis of the target genes of differentially expressed miRNAs in this study indicated that the miRNAs mainly regulated the internal environment of breast tissues through the MAPK signaling pathway. Therefore, further in-depth investigation of milk-derived exosomes is warranted to elucidate the association between miRNA expression profile and milk synthesis microenvironment under HS conditions.

\section{Conclusions}

In conclusion, HS alters the miRNA expression profile of milk exosomes and the differentially expressed miRNAs may play an important role via the MAPK signaling pathway, thereby affecting the survival and lactating function of BMECs in heat- stressed Holstein cows. Therefore, it is crucial to further verify the target genes of milk exosomel miRNAs and associated signaling pathways to provide a theoretical basis for the molecular mechanism by which milk exosomal miRNAs regulate the lactating and physiological cows.

\section{Abbreviations}

BMECs: Bovine mammary epithelial cells; miRNA: microRNA; KEGG: Kyoto Encyclopedia of Genes and Genomes; GO: Gene Onotology; MAPK: Mitogen-activated protein kinase; THI: Temperature and humidity index; HS: Heat stress; Ctr: Contrast; qRT-PCR: Quantitative real-time PCR; RIPA: Radio immune precipitation assay; TSG101: Tumor Susceptibility Gene 101 Protein; NTA: Nanoparticle tracking analysis; TEM: Transmission electron microscopy; PBS: Phosphate buffer solution; SDS: Sodium dodecyl sulfate; TBST: Tris-buffered saline containing

$0.1 \%$ Tween 20

\section{Declarations}

\section{Funding}

This work was supported by the National Natural Science Foundation of China (grant number 32002169) and the Natural Science Foundation of Jiangsu Province (grant number BK20190254).

\section{Conflict of Interest}

The authors declare no conflict of interest. 


\section{Ethics approval and consent to participate}

Not applicable

\section{Consent for publication}

Not applicable

\section{Availability of data and materials}

All data generated or analysed during this study are included in this published article [and its supplementary information files].

\section{Authors' contributions}

Y.W. conceptualized the study, participated in its design and research. J.F. and H.F.Z carried out the molecular studies and sample collection. K.L.C and H.X.L analyzed data and drafted the manuscript. All authors read and approved the final manuscript for publication.

\section{Acknowledgements}

We thank all participants involved in this study.

\section{References}

1. Cai M, Hu Y, Zheng T, He H, Xiao W, Liu B, et al. MicroRNA-216b inhibits heat stress-induced cell apoptosis by targeting Fas in bovine mammary epithelial cells. Cell Stress Chaperones. 2018;23(5):921-31.

2. Sakai S, Hatabu T, Yamamoto Y, Kimura K. Alteration of chemokine production in bovine endometrial epithelial and stromal cells under heat stress conditions. Physiol Rep. 2020;8(22):e14640.

3. Wang Y, Wang HL, Xing GD, Qian Y, Zhong JF, Chen KL. S-allyl cysteine ameliorates heat stressinduced oxidative stress by activating Nrf2/HO-1 signaling pathway in BMECs. Toxicol Appl Pharmacol. 2021;416:115469.

4. Chen KL, Wang HL, Jiang LZ, Qian Y, Yang CX, Chang WW, et al. Heat stress induces apoptosis through disruption of dynamic mitochondrial networks in dairy cow mammary epithelial cells. In Vitro Cell Dev Biol Anim. 2020;56(4):322-31.

5. Liossis SN, Ding XZ, Kiang JG, Tsokos GC. Overexpression of the heat shock protein 70 enhances the TCR/CD3- and Fas/Apo-1/CD95-mediated apoptotic cell death in Jurkat T cells. J Immunol. 1997;158(12):5668-75.

6. Chen W, Wang R, Li D, Zuo C, Wen P, Liu H, et al. Comprehensive Analysis of the Glycome and Glycoproteome of Bovine Milk-Derived Exosomes. J Agric Food Chem. 2020;68(45):12692-701. 
7. Xie MY, Hou LJ, Sun JJ, Zeng B, Xi QY, Luo JY, et al. Porcine Milk Exosome MiRNAs Attenuate LPSInduced Apoptosis through Inhibiting TLR4/NF-kappaB and p53 Pathways in Intestinal Epithelial Cells. J Agric Food Chem. 2019;67(34):9477-91.

8. Zhang S, Chen F, Zhang Y, Lv Y, Heng J, Min T, et al. Recent progress of porcine milk components and mammary gland function. J Anim Sci Biotechnol. 2018;9:77.

9. Zhang M, Ma Z, Li R, Guo S, Qiu Y. X Gao. Proteomic Analysis Reveals Proteins and Pathways Associated with Lactation in Bovine Mammary Epithelial Cell-Derived Exosomes. J Proteome Res. 2020;19(8):3211-9.

10. Yu S, Zhao Z, Sun L, Li P. Fermentation Results in Quantitative Changes in Milk-Derived Exosomes and Different Effects on Cell Growth and Survival. J Agric Food Chem. 2017;65(6):1220-8.

11. Munir J, Lee M, Ryu S. Exosomes in Food: Health Benefits and Clinical Relevance in Diseases. Adv Nutr. 2020;11(3):687-96.

12. Benmoussa A, Lee CH, Laffont B, Savard P, Laugier J, Boilard E, et al. Commercial Dairy Cow Milk microRNAs Resist Digestion under Simulated Gastrointestinal Tract Conditions. J Nutr. 2016;146(11):2206-15.

13. Keller S, Sanderson MP, Stoeck A. P Altevogt. Exosomes: from biogenesis and secretion to biological function. Immunol Lett. 2006;107(2):102-8.

14. Yu T, Zhao C, Hou S, Zhou W, Wang B, Chen Y. Exosomes secreted from miRNA-29b-modified mesenchymal stem cells repaired spinal cord injury in rats. Braz J Med Biol Res. 2019;52(12):e8735.

15. Hoshino A, Costa-Silva B, Shen TL, Rodrigues G, Hashimoto A, Mark MT, et al. Tumour exosome integrins determine organotropic metastasis. Nature. 2015;527(7578):329-35.

16. Luga V, Zhang L, Viloria-Petit AM, Ogunjimi AA, Inanlou MR, Chiu E, et al. Exosomes mediate stromal mobilization of autocrine Wnt-PCP signaling in breast cancer cell migration. Cell. 2012;151(7):154256.

17. Chen J, Chen J, Cheng Y, Fu Y, Zhao H, Tang M, et al. Mesenchymal stem cell-derived exosomes protect beta cells against hypoxia-induced apoptosis via miR-21 by alleviating ER stress and inhibiting p38 MAPK phosphorylation. Stem Cell Res Ther. 2020;11(1):97.

18. Liang C, Liu Y, Xu H, Huang J, Shen Y, Chen F, et al. Exosomes of Human Umbilical Cord MSCs Protect Against Hypoxia/Reoxygenation-Induced Pyroptosis of Cardiomyocytes via the miRNA-1005p/FOX03/NLRP3 Pathway. Front Bioeng Biotechnol. 2020;8:615850.

19. Qiu L, Chen W, Wu C, Yuan Y, Li Y. Exosomes of oral squamous cell carcinoma cells containing miR181a-3p induce muscle cell atrophy and apoptosis by transmissible endoplasmic reticulum stress signaling. Biochem Biophys Res Commun. 2020;533(4):831-7.

20. Wang L, Shi Z, Wang X, Mu S, Xu X, Shen L, et al. Protective effects of bovine milk exosomes against oxidative stress in IEC-6 cells. Eur J Nutr. 2021;60(1):317-27.

21. Ji Z, Liu Z, Chao T, Hou L, Fan R, He R, et al. Screening of miRNA profiles and construction of regulation networks in early and late lactation of dairy goat mammary glands. Sci Rep. 2017;7(1):11933. 
22. Hou J, An X, Song Y, Gao T, Lei Y, Cao B. Two Mutations in the Caprine MTHFR 3'UTR Regulated by MicroRNAs Are Associated with Milk Production Traits. PLoS One. 2015;10(7):e0133015.

23. Title AC, Denzler R, Stoffel M. Uptake and Function Studies of Maternal Milk-derived MicroRNAs. J Biol Chem. 2015;290(39):23680-91.

24. Gonzalez-Rivas PA, Chauhan SS, Ha M, Fegan N, Dunshea FR. RD Warner. Effects of heat stress on animal physiology, metabolism, and meat quality: A review. Meat Sci. 2020;162:108025.

25. Kim KU, Kim WH, Jeong CH, Yi DY, Min H. More than Nutrition: Therapeutic Potential of Breast MilkDerived Exosomes in Cancer. Int J Mol Sci. 2020; 21(19).

26. Zheng Y, Chen KL, Zheng XM, Li HX, Wang GL. Identification and bioinformatics analysis of microRNAs associated with stress and immune response in serum of heat-stressed and normal Holstein cows. Cell Stress Chaperones. 2014;19(6):973-81.

27. van Wijnen AJ, van de Peppel J, van Leeuwen JP, Lian JB, Stein GS, Westendorf JJ, et al. MicroRNA functions in osteogenesis and dysfunctions in osteoporosis. Curr Osteoporos Rep. 2013;11(2):7282.

28. Smythies J, Edelstein L, Ramachandran V. Molecular mechanisms for the inheritance of acquired characteristics-exosomes, microRNA shuttling, fear and stress: Lamarck resurrected? Front Genet. 2014;5:133.

29. Fuziwara CS, ET Kimura. MicroRNAs in thyroid development, function and tumorigenesis. Mol Cell Endocrinol. 2017;456:44-50.

30. Kobayashi Y, Ohtsuki M, Murakami T, Kobayashi T, Sutheesophon K, Kitayama H, et al. Histone deacetylase inhibitor FK228 suppresses the Ras-MAP kinase signaling pathway by upregulating Rap1 and induces apoptosis in malignant melanoma. Oncogene. 2006;25(4):512-24.

31. Li X, Zhou S, Fan T. X Feng. IncRNA DGCR 5/miR27a3p/BNIP3 promotes cell apoptosis in pancreatic cancer by regulating the p38 MAPK pathway. Int J Mol Med. 2020;46(2):729-39.

32. Su C, Huang DP, Liu JW, Liu WY, YO Cao. miR-27a-3p regulates proliferation and apoptosis of colon cancer cells by potentially targeting BTG1. Oncol Lett. 2019;18(3):2825-34.

33. Li H, Lu C, Yao W, Xu L, Zhou J, Zheng B. Dexmedetomidine inhibits inflammatory response and autophagy through the circLrp1b/miR-27a-3p/Dram2 pathway in a rat model of traumatic brain injury. Aging. 2020;12(21):21687-705.

\section{Tables}

Table 1. Sample collection information 


\begin{tabular}{|llll|}
\hline Group & $\begin{array}{l}\text { Average Rectal } \\
\text { temperature } /{ }^{\circ} \mathrm{C}\end{array}$ & $\begin{array}{l}\text { Temperature and humidity } \\
\text { index }(\mathrm{THI})\end{array}$ & $\begin{array}{l}\text { Average breathing rate } \\
(\text { Times } / \mathrm{min})\end{array}$ \\
\hline HS1 & 39.7 & 79.85 & $106 \pm 2.65$ \\
\hline HS2 & 39.8 & 77.99 & $96 \pm 2.00$ \\
HS3 & 40.3 & 80.89 & $108 \pm 2.65$ \\
Ctr1 & 38.1 & 53.30 & $47 \pm 2.03$ \\
Ctr2 & 38.5 & 53.30 & $52 \pm 1.78$ \\
Ctr3 & 38.5 & 54.74 & $53 \pm 2.10$ \\
\hline
\end{tabular}

Table 2. Analysis of sequencing quality

\begin{tabular}{|llllll|}
\hline ID & Raw reads & 3ADT\&length filter & Junk reads & Clean reads & Percentage \\
\hline HS1 & 26374414 & 24093198 & 93407 & 2274910 & 8.63 \\
\hline HS2 & 22752325 & 14132527 & 118346 & 8725710 & 38.34 \\
\hline HS3 & 19778069 & 13537845 & 1902746 & 5301423 & 26.81 \\
\hline Ctr1 & 20620474 & 4013423 & 346546 & 16884173 & 81.88 \\
\hline Ctr2 & 20125725 & 5977011 & 264976 & 14519517 & 72.13 \\
\hline Ctr3 & 24233659 & 12973626 & 122308 & 11553897 & 47.67 \\
\hline
\end{tabular}

Table 3. Rfam, mRNA and Repeats Database Comparison Statistics

\begin{tabular}{|lllllll|}
\hline ID & Clean reads & Rfam & mRNA & Repeats & valid reads & Percentage \\
\hline HS1 & 2274910 & 297854 & 317362 & 54712 & 1604982 & 6.09 \\
\hline HS2 & 8725710 & 1987203 & 458011 & 115812 & 6164684 & 27.09 \\
\hline HS3 & 5301423 & 248341 & 2149666 & 863270 & 2040146 & 10.32 \\
\hline Ctr1 & 16884173 & 2821179 & 705433 & 435686 & 12921875 & 62.67 \\
\hline Ctr2 & 14519517 & 2783887 & 695339 & 477794 & 10562497 & 52.48 \\
\hline Ctr3 & 11553897 & 1962782 & 458834 & 266816 & 8865465 & 36.58 \\
\hline
\end{tabular}


A

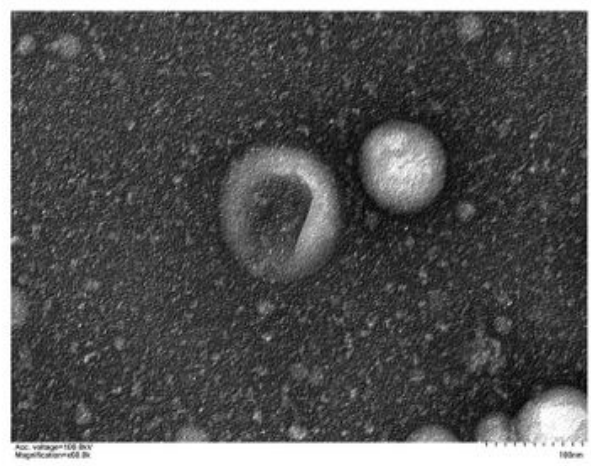

$\mathrm{C}$

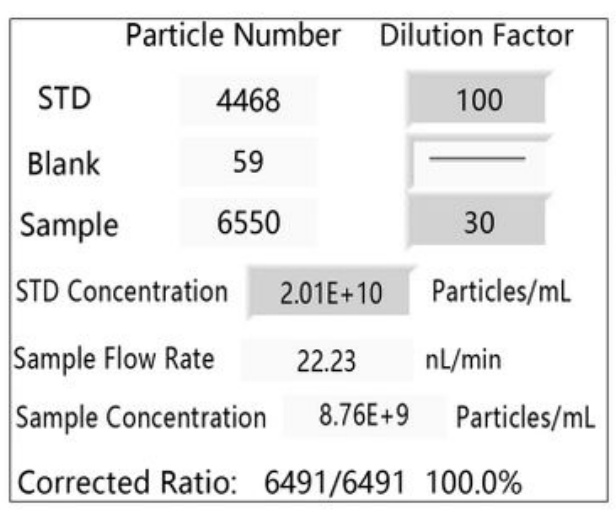

E

\begin{tabular}{|rc|}
\hline Gating Range $30.00-150.00 \mathrm{~nm}$ \\
Total Events 6491 & \\
Gating Events 6348 & \\
\% of all 97.80 & \\
Median 69.25 & $\mathrm{~nm}$ \\
Mean 73.82 & $\mathrm{~nm}$ \\
Std Dev. 17.37 & $\mathrm{~nm}$ \\
\hline
\end{tabular}

B

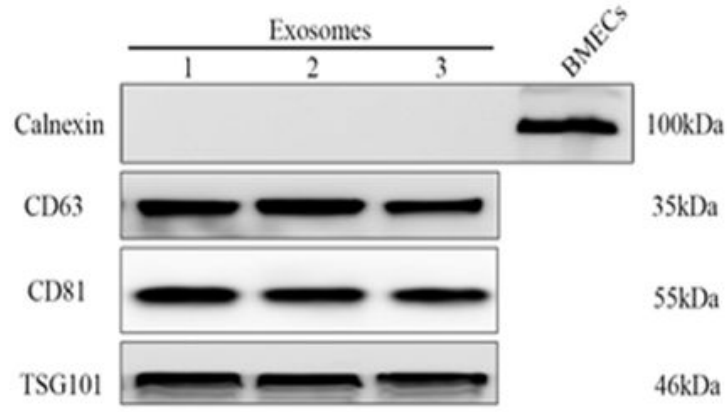

D
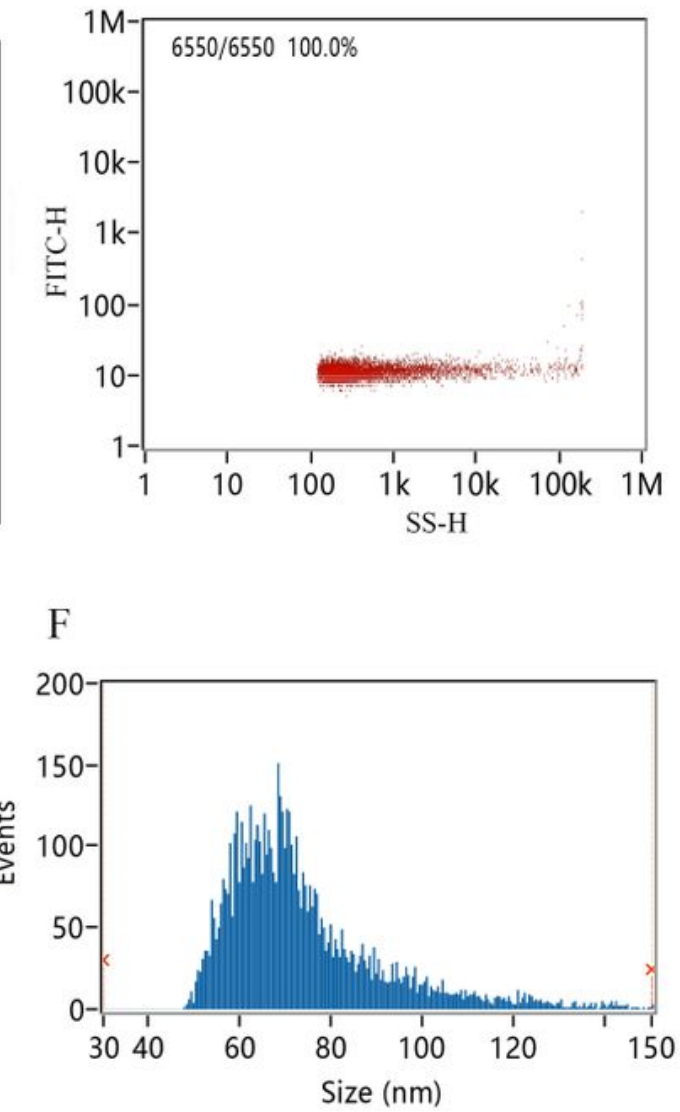

\section{Figure 1}

Exosomes identification. A, Electron micrograph shows the morphology of exosomes from milk. B, Western blotting were used to analyze the expression of CD63, CD81, TSG101, Calnexin. C and D, The concentration of exosomes from milk. E and F, The particle size of exosomes from milk. 
A

B

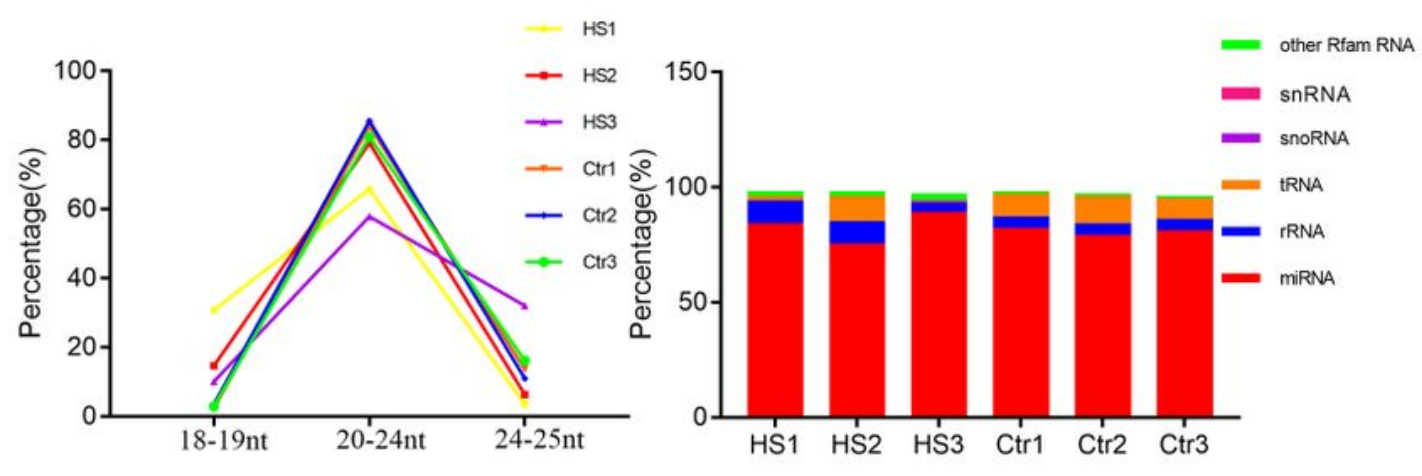

$\mathrm{C}$

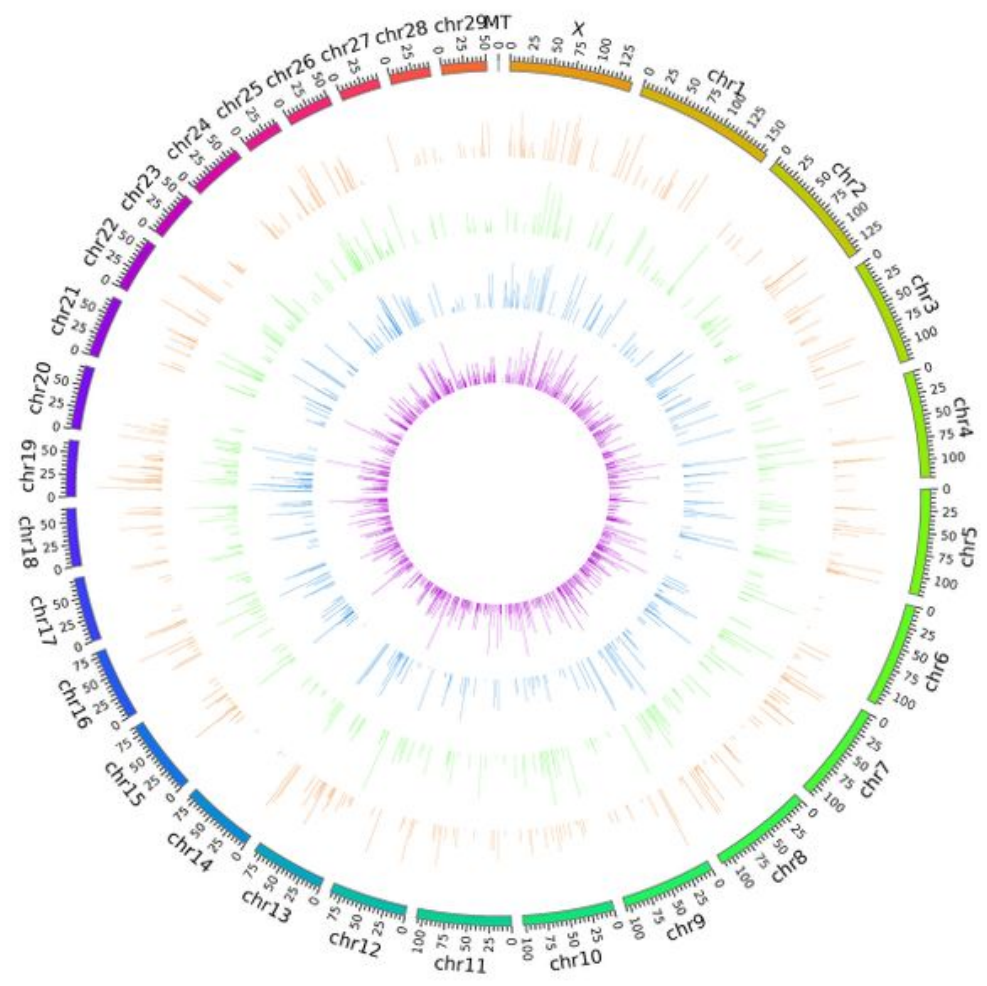

Figure 2

Distribution of miRNAs length, proportion and chromosomal location. A, Ratio of miRNAs fragment length. B, The proportion of non-coding RNA. C, A circle diagram of differentially expressed miRNAs. Outermost ring: known miRNA in Ctr; Secondly: a newly predicted miRNAs in the Ctr; Thirdly, known miRNAs in HS; Innermost circle: a newly predicted miRNAs in the HS. 
A

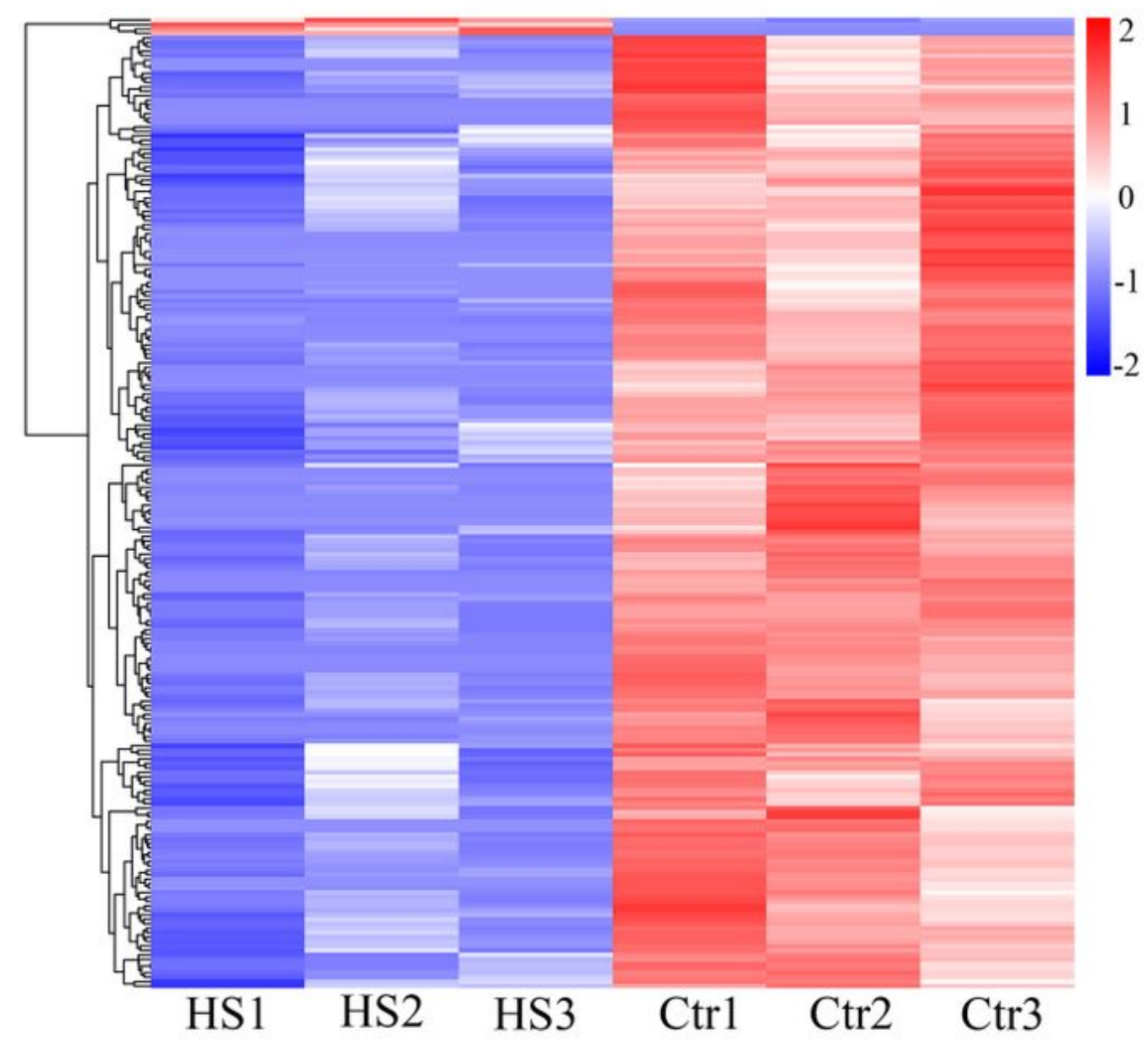

B

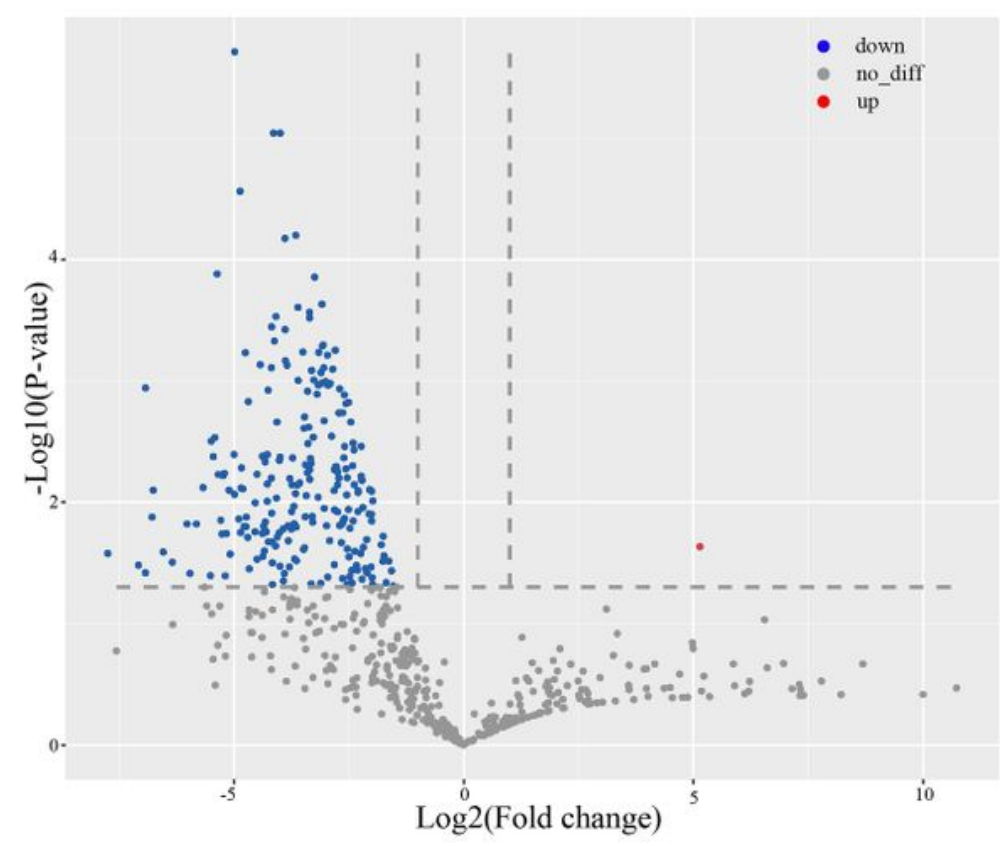

\section{Figure 3}

Heat map and volcanic map analysis of miRNAs differentially expressed by HS and Ctr groups. A, Heat map. The significantly differentially expressed miRNAs in HS vs Ctr. B, Screening for miRNAs involved in HS and Ctr groups. 
A

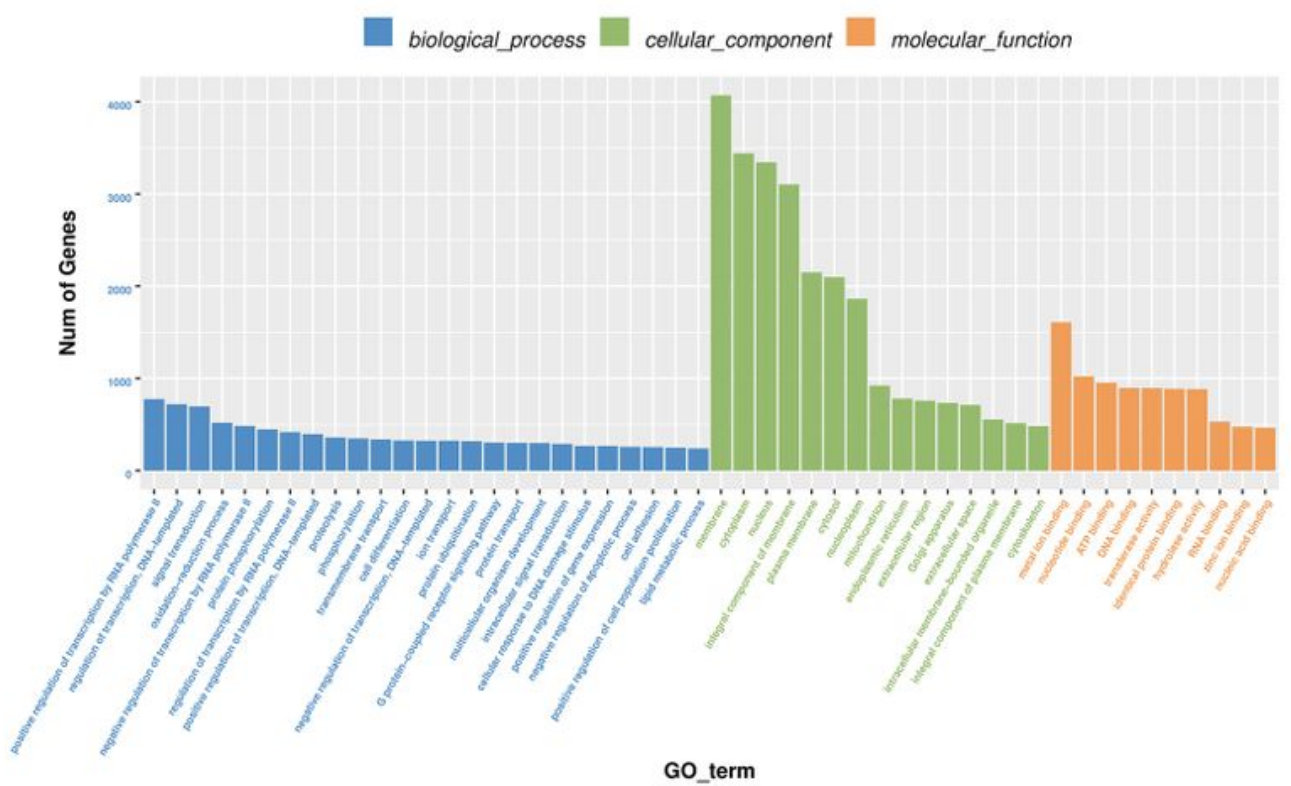

$\mathrm{B}$

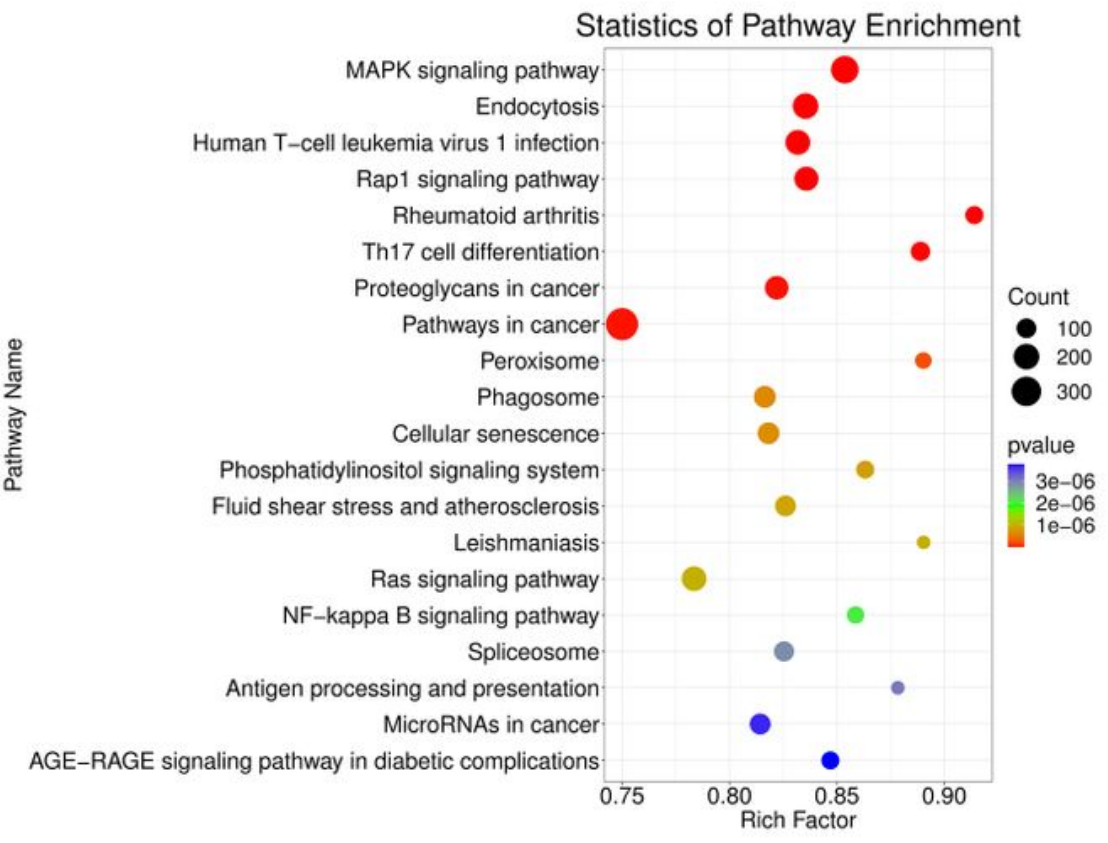

\section{Figure 4}

Enrichment analysis of GO and KEGG pathways with significantly differentially expressed miRNAs. A, GO enrichment, top 30 entry map. Horizontal axis is the $\mathrm{GO}$ entry name and the vertical axis is mum of genes. $B, K E G G$ enrichment, top 20 genes. 
A

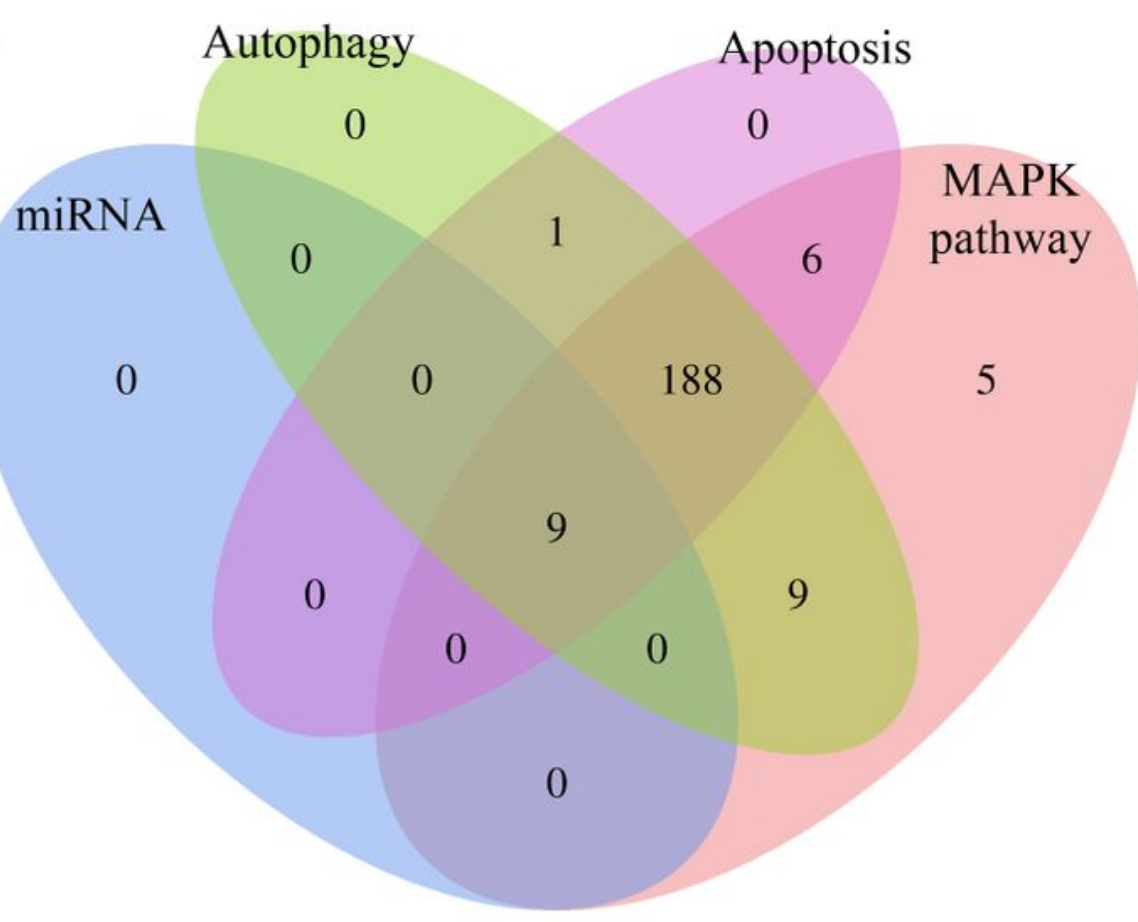

$\mathrm{B}$

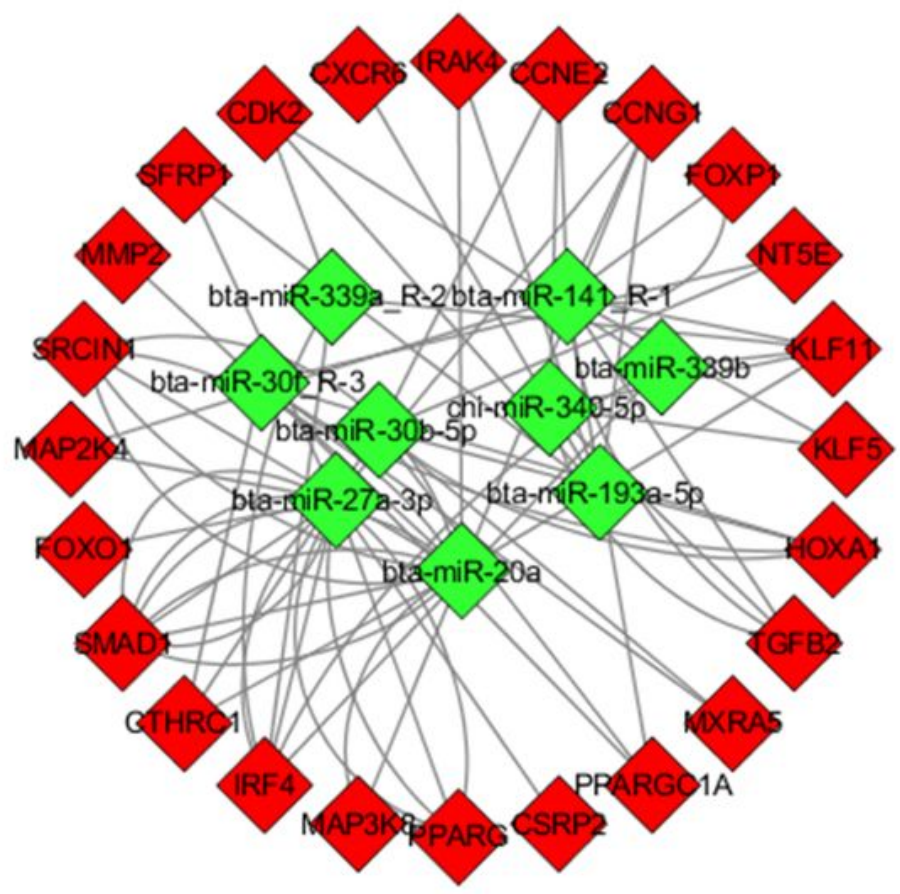

\section{Figure 5}

Screening of differentially expressed miRNAs and their target genes. A, Different miRNA, apoptosis, autophagy, and MAPK signaling pathways of the Venn. B, Network analysis of differentially expressed miRNAs and their target genes in milk. Green rhombus shown in network indicate miRNAs whereas red rhombus their target genes. 


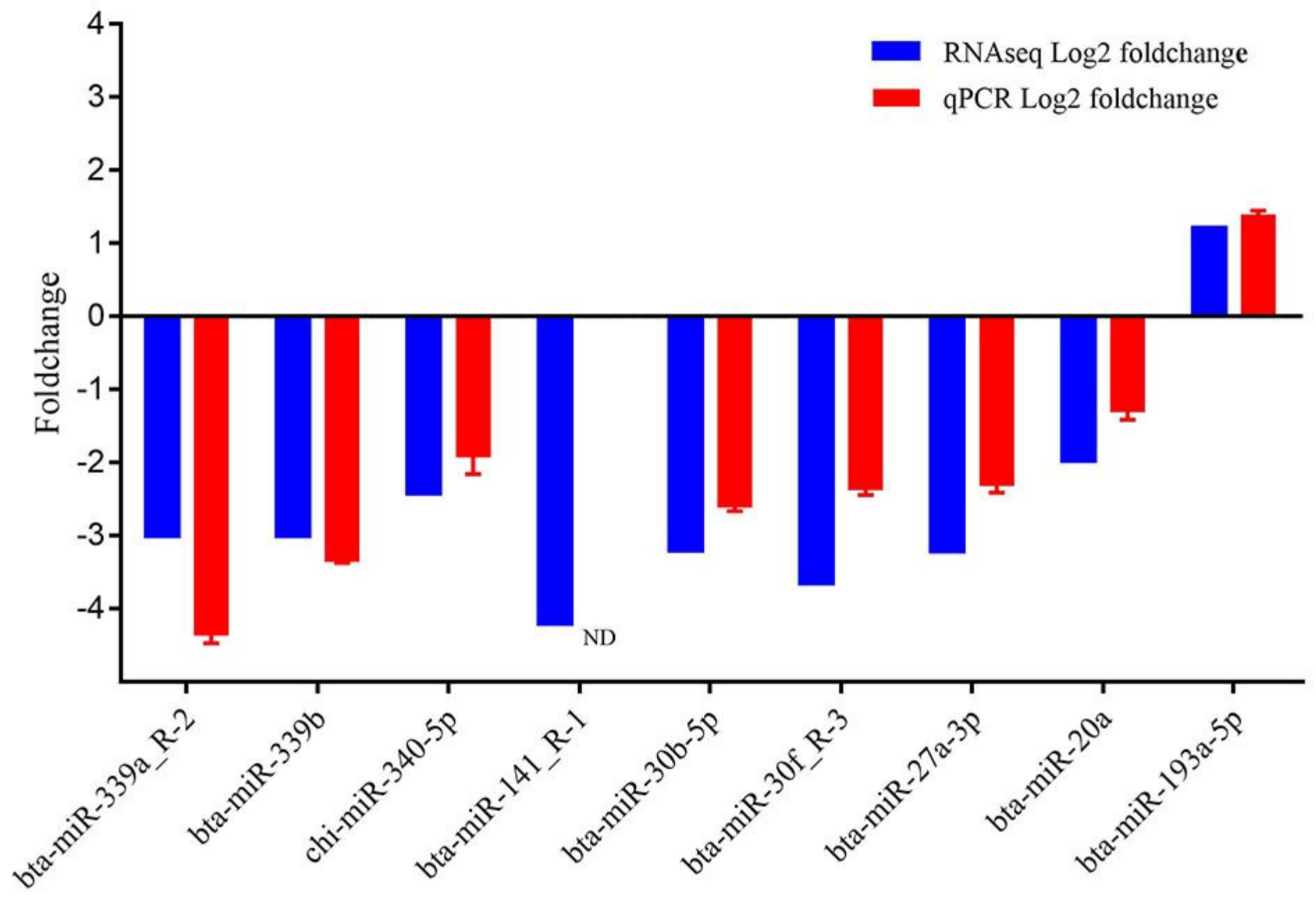

Figure 6

Identification of significantly differentially expressed miRNAs in exosomes. ND means not detected.

\section{Supplementary Files}

This is a list of supplementary files associated with this preprint. Click to download.

- TableS1.docx

- Tables2.docx

- TableS3.docx

- TableS4.docx

- TableS5.xlsx

- TableS6.xlsx

- TableS7.xIsx

- Tables8.xlsx 